Yigit S, McRoberts C, Chakraborty N.

Numerical investigation of laminar Rayleigh-Bénard convection of power-law fluids in square cross-sectional cylindrical annular enclosures. International Communications of Heat and Mass Transfer 2016 DOI: http://dx.doi.org/10.1016/j.icheatmasstransfer.2016.08.025

\section{Copyright:}

(C) 2016. This manuscript version is made available under the CC-BY-NC-ND 4.0 license

DOI link to article:

http://dx.doi.org/10.1016/i.icheatmasstransfer.2016.08.025

Date deposited:

$05 / 08 / 2016$

Embargo release date:

07 September 2017

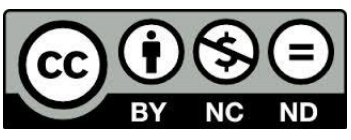

This work is licensed under a

Creative Commons Attribution-NonCommercial-NoDerivatives 4.0 International licence 


\title{
Numerical investigation of laminar Rayleigh-Bénard convection of power-law fluids in square cross-sectional cylindrical annular enclosures
}

\author{
Sahin Yigit ${ }^{1, \bigotimes}$, Calum McRoberts ${ }^{1}$, Nilanjan Chakraborty ${ }^{1}$ \\ ${ }^{1}$ School of Mechanical and Systems Engineering, Newcastle University, Newcastle-Upon-Tyne, NE1 \\ 7RU, UK \\ ${ }^{\square}$ Corresponding author. \\ s.yigit1@ncl.ac.uk
}

KEYWORDS: Natural convection, power-law fluid, cylindrical enclosures, constant wall temperature, constant wall heat flux. 


\section{NOMENCLATURE}

$c_{p}$

$e_{i j}$

$g$

$G r$

h

K

k

L

$n$

$\overline{N u}$

$\overline{N u}_{c y}$

$P$

Pr

$q$

$r$

$r_{i}$

$r_{o}$

$R a$

$T$

$u,(w)$

$U, W$

$U_{\text {ref }}$

$\vartheta$

z

$\alpha$

$\beta$

$\delta, \delta_{t h}$

$\theta$
Specific heat at constant pressure, $\mathrm{J} / \mathrm{kgK}$

Rate of strain tensor, $\mathrm{s}^{-1}$

Gravitational acceleration, $\mathrm{m} / \mathrm{s}^{2}$

Grashof number

Heat transfer coefficient, $\mathrm{W} / \mathrm{m}^{2} \mathrm{~K}$

Consistency, $\mathrm{N} \mathrm{s}$ n $/ \mathrm{m}^{2}$

Thermal conductivity coefficient, W/mK

Height of the enclosure and difference between inner and outer radius, $m$

Power-law index

Mean Nusselt number

Mean Nusselt number for cylindrical annular enclosure

Pressure, $\mathrm{Pa}$

Prandtl number

Heat flux, $\mathrm{W} / \mathrm{m}^{2}$

Radius, $m$

Inner radius, $\mathrm{m}$

Outer radius, $\mathrm{m}$

Rayleigh number

Temperature, $\mathrm{K}$

Radial(vertical) velocity component, $\mathrm{m} / \mathrm{s}$

Dimensionless radial $(U=u L / \alpha)$ and vertical velocity $(W=w L / \alpha)$

Reference velocity scale, $\mathrm{m} / \mathrm{s}$

Characteristic velocity, $\mathrm{m} / \mathrm{s}$

Coordinate in vertical direction, $\mathrm{m}$

Thermal diffusivity, $\mathrm{m}^{2} / \mathrm{s}$

Coefficient of thermal expansion, $1 / \mathrm{K}$

Velocity and thermal boundary-layer thickness, $\mathrm{m}$

Dimensionless temperature

$\begin{array}{cl}\mu & \text { Dynamic viscosity, } \mathrm{Ns} / \mathrm{m}^{2} \\ v & \text { Kinematic viscosity, } \mathrm{m}^{2} / \mathrm{s} \\ \rho & \text { Density, } \mathrm{kg} / \mathrm{m}^{3} \\ \tau_{i j}(\tau) & \text { Stress tensor (stress) } \\ \phi & \text { Azimuthal co-ordinate } \\ \psi & \text { Stream function, } \mathrm{m}^{2} / \mathrm{s} \\ \Psi & \text { Dimensionless stream function }\end{array}$

Subscripts

\begin{tabular}{cl}
\multicolumn{1}{c}{$a$} & Apparent \\
$C$ & Cold wall \\
CWT & Constant wall temperature \\
CWHF & Constant wall heat flux \\
$c e n$ & Geometrical centre \\
$c y$ & Cylindrical Annular Enclosure \\
$e f f$ & Effective value \\
$H$ & Hot wall \\
nom & Nominal value \\
$r e f$ & Reference value \\
$s q$ & Square
\end{tabular}

Special characters

$\Delta T \quad$ Temperature difference, $\mathrm{K}$

$\Delta_{\text {min,cell }} \quad$ Minimum cell distance, $\mathrm{m}$

\section{ABSTRACT}

Steady-state laminar Rayleigh-Bénard convection (i.e. differentially heated horizontal walls heated from below) of power-law fluids in square cross-sectional cylindrical annular enclosures has been numerically investigated under the assumption of axisymmetry. The numerical simulations have been conducted for a range of different values of nominal Rayleigh number $R a$, nominal Prandtl number $\operatorname{Pr}$, power-law index $n$ and internal radius to enclosure height ratio $r_{i} / L$ (i.e. $10^{3} \leq R a \leq 10^{5} ; 10 \leq \operatorname{Pr} \leq 10^{4} ; 0.6 \leq n \leq 1.8 ; 0 \leq r_{i} / L \leq 24$ ) for both constant wall temperature (CWT) and constant wall heat flux (CWHF) boundary conditions for differentially heated horizontal walls. It has been found that convective transport is stronger for CWT boundary condition than for CWHF boundary condition for large (small) values of $R a(n)$ for a given set of values of $n(R a), P r$, and $r_{i} / L$, but an opposite trend is observed for small (large) values of $R a(n)$. The mean Nusselt number $\overline{N u}_{c y}$ does not show a monotonic increase with increasing (decreasing) $R a(n)$ especially for small values of $r_{i} / L$ for a given value of $\operatorname{Pr}$ due to changes in flow patterns (i.e. number of convection cells). However, the mean Nusselt number $\overline{N u}_{c y}$ and flow patterns for large values of $r_{i} / L$ approach those for square enclosures $\left(r_{i} / L \rightarrow \infty\right)$ for both CWT and CWHF boundary conditions. Additionally, the critical Rayleigh number $R a_{\text {crit }}$ for the onset of convection has been found to be mostly insensitive to the value of $r_{i} / L$ for both CWT and CWHF boundary conditions. 


\section{INTRODUCTION}

Natural convection of non-Newtonian fluids in enclosed spaces is relevant to many engineering applications (i.e. food and chemical industries, solar power systems and cooling of electronic devices). Thus, perhaps unsurprisingly, natural convection of non-Newtonian fluids obeying power-law model of viscosity has been a subject of several previous analyses (see Table 1 of Ref. [1]) and an enhancement of convective heat transfer rate has been reported for shear thinning fluids [2-6] (also see references in Ref. [1]). Most of these analyses have been carried out for rectangular enclosures but cylindrical enclosures are more relevant to practical applications (e.g. solar collectors, heat exchangers etc.). The effects of ratio of the internal radius to enclosure height (i.e. $r_{i} / L$ ) on natural convection of power-law fluids in cylindrical annular enclosures with differentially heated vertical walls have recently been investigated by Yigit et al. [7,8]. It has been found that convective transport strengthens with increasing $r_{i} / L$ for natural convection of power-law fluids in cylindrical annular spaces with differentially heated vertical walls. However, the effects of $r_{i} / L$ on natural convection of power-law fluids in Rayleigh-Bénard configuration (i.e. differentially heated horizontal walls with higher bottom wall temperature) have not been analysed in open literature. It is important to note that convection sets in once a finite temperature difference is established between the active walls in differentially heated vertical wall configuration, whereas a critical Rayleigh number needs to be surpassed for the onset of fluid motion in the Rayleigh-Bénard configuration. Therefore, steady state laminar Rayleigh-Bénard convection of power-law fluids in square cross-sectional cylindrical annular enclosures has been investigated in this study to address this deficit in existing literature. Here, the simulations have been carried out for a range of different values of nominal Rayleigh (e.g. $R a=10^{3}-10^{5}$ ), Prandtl (e.g. $\left.\operatorname{Pr}=10-10^{4}\right)$ numbers and power-law indexes $(n=0.6-1.8)$ for different values of cylinder radius (e.g. $r_{i} / L=0-24$ where $r_{i}$ and $L$ are the inner radius and the cylinder height respectively). In this respect, the main objectives of this analysis are to demonstrate the effects of $R a, P r, n$ on steady-state laminar RayleighBénard convection of power-law fluids in square cross-sectional cylindrical annular enclosures for different values of $r_{i} / L$, and provide physical explanations for the observations based on numerical findings.

\section{MATHEMATICAL BACKGROUND}

The viscous stress tensor $\tau_{i j}$ for a power-law fluid can be expressed as: $\tau_{i j}=\mu_{a} e_{i j}=K\left(e_{k l} e_{k l} / 2\right)^{(n-1) / 2} e_{i j}$ where $e_{i j}=\left(\partial u_{i} / \partial x_{j}+\partial u_{j} / \partial x_{i}\right)$ is the rate of strain tensor, $K$ is the consistency, $n$ is the power-law index and $\mu_{a}=K\left(e_{k l} e_{k l} / 2\right)^{(n-1) / 2}$ is the apparent viscosity. The apparent viscosity $\mu_{a}$ decreases (increases) with increasing shear rate for $n<1 \quad(n>1)$ and thus fluids with $n<1 \quad(n>1)$ are referred to as shear-thinning (shear-thickening) fluids, whereas $n=1$ represents Newtonian fluids. The present analysis is conducted in nondimensional form for the purpose of generalization where the spatial co-ordinates, velocity components, pressure, shear stress and temperature are non-dimensionalised in the following manner:

$r_{i}^{+}=r_{i} / L, z^{+}=z / L, u_{i}{ }^{+}=u_{i} / U_{r e f}, P^{+}=P / \rho U_{r e f}{ }^{2}, \tau_{i j}{ }^{+}=\tau_{i j} L / \rho \alpha U_{r e f}, \Theta=\left(T-T_{r e f}\right) / \Delta T_{r e f}$

where $U_{r e f}$ is given by: $U_{r e f}=\alpha / L$ according to several previous analyses [1,2,6-8], $T_{r e f}$ is the reference temperature and $\Delta T_{r e f}$ is the reference temperature difference. Here, $\Delta T_{r e f}$ is taken to be $\left(T_{H}-T_{C}\right)(q L / k)$ for CWT (CWHF) configurations. Moreover, $T_{r e f}$ is taken to be the cold wall temperature $T_{c}$ for the CWT configuration and the temperature at the geometrical centre of the annular enclosure (i.e. $T_{r e f}=T_{c e n}$ ) for the CWHF boundary condition. This yields the following non-dimensional forms of conservation equations for power-law fluids with temperature-independent thermo-physical properties under the assumption of axisymmetry: Non-dimensional mass conservation equation:

$\left(1 / r^{+}\right) \partial\left(r^{+} u^{+}\right) / \partial r^{+}+\left(\partial w^{+} / \partial z^{+}\right)=0$

Non-dimensional momentum conservation equations

Radial direction:

$\widehat{u^{+}\left(\partial u^{+} / \partial r^{+}\right)+} w^{+}\left(\partial u^{+} / \partial z^{+}\right)=-\partial P^{+} / \partial r^{+}+\left(1 / r^{+}\right) \partial\left(r^{+} \tau_{r r}{ }^{+}\right) / \partial r^{+}-\tau_{\phi \phi}{ }^{+} / r^{+}+\partial\left(\tau_{r z}{ }^{+}\right) / \partial z^{+}$

Vertical direction:

$\overline{u^{+}\left(\partial w^{+} / \partial r^{+}\right)+w^{+}}\left(\partial w^{+} / \partial z^{+}\right)=-\partial P^{+} / \partial z^{+}+\operatorname{RaPr} \Theta+\left(1 / r^{+}\right) \partial\left(r^{+} \tau_{r z}{ }^{+}\right) / \partial r^{+}+\partial\left(\tau_{z z}{ }^{+}\right) / \partial z^{+}$

Non-dimensional energy conservation equation:

$u^{+}\left(\partial \Theta / \partial r^{+}\right)+w^{+}\left(\partial \Theta / \partial z^{+}\right)=\left(1 / r^{+}\right) \partial\left(r^{+} \partial \Theta / \partial r^{+}\right) / \partial r^{+}+\partial^{2} \Theta / \partial z^{+} \partial z^{+}$

where $r$ is the radial co-ordinate and $z$ axis is taken to align with the vertical direction, whereas the axisymmetric flow is assumed to be independent of the azimuthal direction $\phi$ as schematic diagram of the configuration is shown in Fig.1. In Eq. 2, $R a$ and $P r$ are the nominal Rayleigh and Prandtl numbers, which are defined for the CWT (CWHF) boundary conditions using the reference temperature differences $\left(T_{H}-T_{c}\right)(q L / k)$ in the following manner:

$R a_{C W T}=g \beta\left(T_{H}-T_{C}\right) L^{2 n+1} / \alpha^{n}(K / \rho) ; R a_{C W H F}=g \beta q L^{2 n+2} / \alpha^{n} k(K / \rho) ; \operatorname{Pr}=(K / \rho) \alpha^{n-2} L^{2-2 n}$ 
The local heat transfer coefficient $h$ is defined as:

$h=\left|-k(\partial T / \partial z)_{z=0} \times 1 /\left(T_{z=0}-T_{z=L}\right)\right|$

The mean heat transfer coefficient $\bar{h}$ and the mean Nusselt number $\overline{N u}_{c y}$ are evaluated as:

$\bar{h}=\int_{r_{i}}^{r_{i}+L} 2 \pi r h d r /\left[\pi\left(r_{i}+L\right)^{2}-\pi r_{i}^{2}\right], \overline{N u}_{c y}=\bar{h} L / k$

Using Buckingham's pi theorem, it is possible to show that the Nusselt number for natural convection of powerlaw fluids in square cross-sectional cylindrical annular enclosures can be expressed as: $N u=f\left(\operatorname{Ra}, \operatorname{Pr}, n, r_{i} / L\right)$. Equations 2i-iv are solved in a coupled manner in conjunction with the following boundary conditions. The two vertical walls are kept under adiabatic conditions (i.e. $\partial \Theta / \partial r^{+}=0$ at $r^{+}=r_{i} / L$ and $r^{+}=r_{i} / L+1$ ), and both velocity components (i.e. $u^{+}$and $w^{+}$) are identically zero on each boundary because of the no-slip condition and impenetrability of rigid walls. For the CWHF configuration, the heat fluxes for horizontal hot and cold walls are specified (i.e. $-\partial \Theta / \partial z^{+}=1$ at $z^{+}=0.0$ and $z^{+}=1.0$ respectively). By contrast, the temperatures of horizontal walls are specified (i.e. $\Theta=1$ and $\Theta=0$ at $z^{+}=0.0$ and $z^{+}=1.0$ respectively) for CWT configuration.

\section{NUMERICAL IMPLEMENTATION}

The governing equations (Eqs. 2i-iv) are solved in the axisymmetric cylindrical coordinates using the finitevolume methodology. The present numerical scheme has been validated for natural convection of both Newtonian and power-law fluids in square enclosures. Interested readers are referred to Refs. [1,6-8] for further information of numerical procedure and benchmarking and grid independence analysis. It has been ensured that a change in the computational grid did not lead to a change in flow pattern within the enclosure during the course of grid independency analysis [1]. Minimum and maximum levels of $\mu$ are assumed to be $\mu_{\min }=10^{-4} \mu_{n=1}$ and $\mu_{\max }=$ $10^{4} \mu_{n=1}$ respectively where $\mu_{n=1}$ is the viscosity of the Newtonian fluid for the same nominal values of Rayleigh and Prandtl numbers. It has been checked that the results remain independent of the choices of $\mu_{\min }$ and $\mu_{\max }$ beyond these values.

Previous analyses [1,9] reported multiple steady two-dimensional Newtonian (i.e. $n=1$ ) flow patterns in the super-critical regime (when the critical Rayleigh number for linear instability is surpassed) of Rayleigh-Bernard convection for square (i.e. $A R=1$ ) enclosures. Furthermore, one may obtain multiple solutions for RayleighBernard convection in the case of shear-thinning (i. e. $n<1$ ) fluids when the power-law exponent $n$ is smaller than a threshold value [10]. Furthermore, recent numerical investigations in the case of rectangular enclosures suggested that the simulations for both Newtonian and power-law fluids in Rayleigh-Bernard configuration can be sensitive to the initial conditions $[9,10]$. For the above reasons, the simulations in the current analysis have been carried out for two types of initial conditions. In one set of simulations, the quiescent flow condition is used for the initial condition of Newtonian fluid simulations, whereas the steady state simulation results obtained for a smaller value of $R a$ is used for the initial condition for the cases with $R a \geq 1 \times 10^{3}$ in the second method. These two methodologies will henceforth be referred to as the "quiescent flow (Q.F.)" and "established flow (E.F.)" initial conditions respectively, in this paper. The steady-state solution obtained for Newtonian (i.e. $n=1.0$ ) fluids based on E.F. initial condition for a given set of values of $R a$ and $P r$ has in turn been used as an initial condition for one set of simulations involving non-Newtonian power-law fluids (i.e. E.F. initial condition for power-law fluids), whereas the quiescent condition is used as an initial condition (i.e. Q.F. initial condition for power-law fluids) for the other set of power-law fluid simulations.

\section{SCALING ANALYSIS}

A detailed scaling analysis is performed to estimate effects of $R a, P r, n, r_{i} / L$ on laminar Rayleigh-Bénard convection regime of power-law fluids in current study. Equating the order of magnitudes of inertial and buoyancy terms yields $w \sim \sqrt{g \beta\left(T_{H}-T_{C}\right) L}\left(w \sim \sqrt{g \beta q \delta_{t h} L / k}\right)$ can be obtained for CWT (CWHF) configurations. Based on the continuity equation for axisymmetric geometry with $r_{i} \neq 0$ leads to:

$\frac{1}{r} \frac{\partial(r u)}{\partial r} \sim\left(\frac{u}{r}+\frac{u}{L}\right) \sim \frac{\partial w}{\partial z} \sim \frac{w}{L}$

which leads to the following relation based on scaling estimate of $u$ :

$u \sim \frac{w}{\left(1+L / r_{i}\right)} \sim \frac{\alpha}{L} \frac{\sqrt{R a_{C W T} P r}}{\left(1+L / r_{i}\right)} ; u \sim \frac{w}{\left(1+L / r_{i}\right)} \sim \frac{\alpha}{L}\left(R a_{C W H F}{ }^{\left(\frac{n}{2}+\frac{1}{2}\right) /\left(\frac{n}{2}+2\right)} \operatorname{Pr}^{\left(\frac{n}{2}+1\right) /\left(\frac{n}{2}+2\right)} f_{2}{ }^{-\left(\frac{n}{2}+\frac{1}{2}\right) /\left(\frac{n}{2}+1\right)}\right) /\left(1+L / r_{i}\right)$

Similarly, equating the order of magnitudes of inertial and viscous terms in the vertical (radial) direction (i.e. $\rho w^{2} / L \sim\left[(K / \delta)(w / \delta)^{n}\right]\left(\rho u^{2} / L \sim\left[\left(K / \delta_{1}\right)\left(u / \delta_{1}\right)^{n}\right]\right)$, it is possible to estimate hydrodynamic boundary layer thickness in the following manner for $r_{i} \neq 0$ where $\delta\left(\delta_{1}\right)$ is the hydro-dynamic boundary layer thickness on the vertical (horizontal) walls respectively:

$\left.\delta \sim L /\left(\operatorname{Ra}_{C W T}{ }^{2-n} \operatorname{Pr}^{-n}\right)^{\frac{1}{2(n+1)}}\right), \delta \sim L /\left(R a_{C W H F}{ }^{1-n / 2} \operatorname{Pr}^{-n / 2}\right)^{1 /\left(\frac{n}{2}+2\right)} f_{2}{ }^{(n / 2-1) /\left(\frac{n}{2}+2\right)}$ 
$\delta_{1} \sim L\left(1+L / r_{i}\right)^{(2-n) /(n+1)} /\left(\operatorname{Ra}_{C W T}{ }^{2-n} \operatorname{Pr}^{-n}\right)^{\frac{1}{2(n+1)}}(\mathrm{CWT})$,

$\delta_{1} \sim L\left(1+L / r_{i}\right)^{(2-n) /(n+1)} /\left(\operatorname{Ra}_{C W H F}{ }^{1-n / 2} \operatorname{Pr}^{-n / 2}\right)^{1 /\left(\frac{n}{2}+2\right)} f_{2}{ }^{(n / 2-1) /\left(\frac{n}{2}+2\right)} \quad(\mathrm{CWHF})$

Using Eq. 8, it is possible to estimate the effective viscosity in the vertical (horizontal) boundary layers (i.e. $\mu_{e f f}{ }^{V} \sim K(w / \delta)^{n-1}\left(\mu_{e f f}{ }^{H} \sim K\left(u / \delta_{1}\right)^{n-1}\right)$ in order to predict the effective Rayleigh numbers for $r_{i} \neq 0$ in the vertical and horizontal boundary layers in the following manner:

$R a_{C W T, e f f}{ }^{V}=\rho^{2} c_{p} g \beta\left(T_{H}-T_{C}\right) L^{3} / \mu_{e f f}{ }^{V} k \sim R a^{(5-n) /(2 n+2)} \operatorname{Pr}^{(1-n) /(2 n+2)}$

$R a_{C W H F, e f f}{ }^{V}=\rho^{2} c_{p} g \beta q L^{4} / \mu_{e f f} V k^{2} \sim R a^{(7-2 n) /(n+4)} \operatorname{Pr}^{(2-2 n) /(n+4)}$

$R a_{C W T, \text { eff }}{ }^{H}=\rho^{2} c_{p} g \beta\left(T_{H}-T_{C}\right) L^{3} / \mu_{e f f}{ }^{H} k \sim R a^{(5-n) /(2 n+2)} \operatorname{Pr}^{(1-n) /(2 n+2)}\left(1+L / r_{i}\right)^{3(n-1) /(n+1)}$

$R a_{C W H F, \text { eff }}{ }^{H}=\rho^{2} c_{p} g \beta q L^{4} / \mu_{e f f}{ }^{H} k^{2} \sim R a^{(7-2 n) /(n+4)} \operatorname{Pr}^{(2-2 n) /(n+4)}\left(1+L / r_{i}\right)^{3(n-1) /(n+1)}$

Equations 9 and 10 show that the effective value of Rayleigh number $R a_{\text {eff }}$ increases (decreases) with decreasing (increasing) values of $n$ due to weaken (strengthen) viscous resistance as a result of shear thinning (thickening) behaviour. It is also can be seen $R a_{C W T, e f f}{ }^{H}$ and $R a_{C W H F, \text { eff }}{ }^{H}$ decrease with decreasing $r_{i} / L$ for shear thinning fluids, but an opposite behaviour can be expected for shear thickening fluids (i.e. $n>1$ ). Also, $R a_{e f f} V$ and $R a_{\text {eff }}{ }^{H}$ remain of the same order of magnitude for two dimensional square enclosures $\left(r_{i} \rightarrow \infty\right)$ since velocity scales and boundary layer thicknesses remain same order in both directions. It is worth noting that in the current analysis the $R a_{\text {eff }}$ (see Eqs. 9-10) becomes increasingly larger than its nominal value for decreasing values of $n$ and $P r$ for shear thinning fluids (i.e. $<<1$ ). The scaling relations given by Eqs. $6-10$ need to be considered with caution because using $r_{i}=0$ leads to singularities so these relations need to be treated in an order of magnitude sense for explaining the trends observed from simulation results.

\section{RESULTS AND DISCUSSION}

\subsection{Effects of nominal Rayleigh number $R a$, power-law index $n$ and Prandtl number $P r$}

The variation of $\overline{N u} c y$ with $\operatorname{Pr}$ for different values of $R a, n, r_{i} / L$ and different initial conditions (i.e. E.F. and Q.F.) is shown in Figs. 2-3 with representative flow patterns in the case of CWT and CWHF boundary conditions, respectively. Figures 2-3 indicate that $\overline{N u}_{c y}$ increases (decreases) monotonically with increasing $R a(n)$ for shear thickening fluids (i.e. $n>1$ ) and similar flow patterns are observed regardless of the choices of boundary and initial conditions. By contrast, the variation of $\overline{N u}_{c y}$ shows a non-monotonic trend with an increase in $R a$ due to changes in flow patterns for Newtonian (i.e. $n=1$ ) and shear thinning fluids (i.e. $n<1$ ) for a given set of values of $\operatorname{Pr}$ and $r_{i} / L$ for both choices of initial and boundary conditions. Although convective heat transfer is expected to strengthen with increasing $R a$, a change in the streamline pattern modifies the distance between isotherms in the vicinity of hot and cold walls, which in turn leads to a non-monotonic variation of $\overline{N u}_{c y}$ with $R a$ for both Newtonian (i.e. $n=1$ ) and shear thinning fluids (i.e. $n<1$ ) for a given set of values of $\operatorname{Pr}$ and $r_{i} / L$. This effect is particularly strong for a combination of large values of $R a$ and small values of $n$ and $P r$. Equations 9 and 10 indicate that the effective Rayleigh number $R a_{\text {eff }}$ becomes increasingly larger than its nominal value for decreasing values of $n$ and $P r$ for shear thinning fluids (i.e. $n<1$ ). Thus, steady state solution corresponding to a particular type of flow pattern such as one cell structure, which is obtained for small values of $R a_{e f f}$, may not be possible to obtain for large values of $R a_{e f f}$. Thus, steady state solution may exhibit multiple cell patterns for large values of $R a_{\text {eff }}$ but multiple cell structure acts to reduce the mean Nusselt number.

Furthermore, the variations of the $\overline{N u}_{c y}$ and the streamline pattern are found to be insensitive to the choices of $P r$ and initial condition for both Newtonian (i.e. $n=1$ ) and shear thickening fluids (i.e. $n>1$ ), whereas the influences of $P r$ and initial condition are found to be significant in the case of shear thinning fluids (i.e. $n<1$ ) for a given set of values of $R a$ and $r_{i} / L$ for both CWT and CWHF configurations. The thermal boundary layer remains much thinner than the hydro-dynamic boundary layer for $\operatorname{Pr}>>1$ so the relative balance between the buoyancy and viscous forces is principally affected by nominal Prandtl number [6-8,11], which does not alter the thermal transport within the thermal boundary layer for Newtonian fluids and shear-thickening fluids. This gives rise to a weak $\mathrm{Pr}$ dependence of $\overline{N u}_{c y}$ for Newtonian fluids and shear-thickening fluids. Scaling estimates given by Eqs. $8 \mathrm{i}$ and $8 \mathrm{ii}$ suggest that the thickness of hydrodynamic boundary layer decreases with decreasing (increasing) $n(R a)$. Thus, both hydrodynamic and thermal boundary layers remain thin for a combination of high nominal Rayleigh number and small power-law exponent, and therefore a change in $\operatorname{Pr}$ alters the thermal boundary layer thickness and in turn $\overline{N u}_{c y}$ significantly.

\subsection{Effects of $r_{i} / L$}


The variations of $\overline{N u}_{c y}$ with $r_{i} / L$ for different $R a, n$, and initial conditions (i.e. E.F. and Q.F.) at $P r=10^{3}$ are shown in Fig. 4-5 with representative flow patterns in the CWT and CWHF configurations for $0 \leq r_{i} / L \leq 1$ and $1 \leq r_{i} / L \leq 24$ respectively. It can be seen from Figs. $4-5$ that the values of $\overline{N u}_{c y}$ and flow patterns are not affected by the modification of $r_{i} / L$ for shear thickening fluids (i.e. $n>1$ ) irrespective of the choices of initial and boundary conditions. However, $\bar{N} \bar{u}_{c y}$ shows a non-monotonic variation with $r_{i} / L$ due to changes in flow pattern for both Newtonian (i.e. $n=1$ ) and shear thinning fluids (i.e. $n<1$ ) for a given set of values of $R a$ and $P r$ irrespective of the choices of initial and boundary conditions. This tendency is prevalent for small values of $r_{i} / L$ for cases with a combination of high values of $R a$ and small values of $n$. Equations 9 and 10 indicate that the effective Rayleigh number $R a_{e f f}$ becomes increasingly larger than its nominal value for increasing values of $r_{i} / L$ for shear thinning fluids (i.e. $<<1$ ). Thus, steady state solution corresponding to a particular type of flow pattern associated with small values of $R a_{e f f}$, may not be possible to obtain for large values of $R a_{e f f}$, where the steady state solution yields a different flow pattern. The flow patterns have been found to be strongly dependent on initial condition especially for shear thinning fluids (i.e. $n<1$ ) for both CWT and CWHF boundary conditions. Although $\overline{N u}_{c y}$ exhibits a non-monotonic variation with $r_{i} / L$ for small values of $r_{i} / L$, the mean Nusselt number $\overline{N u}_{c y}$ and flow pattern for large values of $r_{i} / L$ approach those for square enclosures $\left(r_{i} / L \rightarrow \infty\right)$ irrespective of the choice of initial and boundary conditions.

Moreover, the mean Nusselt number $\overline{N u}_{c y}$ assumes higher values for CWT boundary condition than for CWHF boundary condition for large (small) values of $R a(n)$ for a given set of values of $n(R a), P r, n$ and $r_{i} / L$ but an opposite trend is observed for small (large) values of $R a(n)$ as long as flow pattern does not change with the alternation of $R a$ and $n$. This behaviour can be explained in the following manner. The wall heat flux on the horizontal wall $q \sim h \Delta T$ can be scaled as: $q \sim h \Delta T \sim k \Delta T / \delta_{t h} \sim k\left(\Delta T / \delta_{1}\right) f$, so the $\overline{N u}_{c y}$ can be scaled as: $\overline{N u}_{c y} \sim h L / k \sim q L / \Delta T k \sim L / \delta_{t h} \sim\left(L / \delta_{1}\right) f$, where $f\left(R a, \operatorname{Pr}, n, r_{i} / L\right)$ is the function which accounts for the ratio of hydro-dynamic to thermal boundary layer thickness (i.e. $f \sim \delta_{1} / \delta_{t h}$ ). Using Eqs. $8 \mathrm{i}$ and $8 \mathrm{ii}$ one obtains:

$\overline{N u}_{c y} \sim f_{C W T}\left(R a_{C W T}{ }^{2-n} \operatorname{Pr}^{-n}\right)^{\frac{1}{2(n+1)}}\left(1+L / r_{i}\right)^{(n-2) /(n+1)}$;

$\overline{N u}_{c y} \sim f_{C W H F}{ }^{(n+1) /\left(\frac{n}{2}+2\right)}\left(R a_{C W H F}{ }^{1-n / 2} \operatorname{Pr}^{-n / 2}\right)^{1 /\left(\frac{n}{2}+2\right)}\left(1+L / r_{i}\right)^{(n-2) /(n+1)}$

Eq. 11 shows that the exponent of $R a_{C W T}$ (i.e. $\left.(2-n) /(2 n+2)\right)$ assumes greater than exponent of $R a_{C W H F}$ (i.e. $(2-n) /(n+4))$ for the range of $n$ considered here. This difference widens with decreasing $n$. By contrast, the exponent of $\mathrm{Pr}$ assumes greater negative value for CWT boundary condition (i.e. $-n /(2 n+2))$ than the corresponding value in the case of CWHF boundary condition (i.e. $-n /(n+4))$. The effects of higher exponent of nominal Rayleigh number dominate over the influences of higher magnitude of negative exponent of Prandtl number for high (small) values of $R a(n)$, which leads to higher values of mean Nusselt number $\overline{N u} \bar{c}_{c y}$ in the CWT configuration than in the case of CWHF boundary condition provided the flow pattern remains unchanged. By contrast, the effects of higher magnitude of negative exponent of Prandtl number overcome the influences of higher exponent of nominal Rayleigh number for small (high) values of $R a(n)$, which leads to higher values of mean Nusselt number $\overline{N u}_{c y}$ in the CWHF configuration than in the case of same flow pattern under CWT boundary condition. Furthermore, Eqs. $11 \mathrm{i}$ and $11 \mathrm{ii}$ indicate that $\overline{N u}_{c y}$ is expected to increase with increasing $r_{i} / L$ for the range of values of $n$ considered here provided $R a, P r, n$ and the flow pattern remain unchanged.

Turan et al. [6] utilized the scaling estimates given by Eqs. 11i-ii to propose correlations for the mean Nusselt number for natural convection of power-law fluids in square enclosures (i.e. $L / r_{i}=0$ ), which is modified here for better accuracy for shear-thickening (i.e. $n>1$ ) fluids, and these correlations are presented in Table 1. It is worth noting that the correlations given in Table 1 are only valid for one cell flow pattern. Although the non-monotonic variation of $\overline{N u}_{c y}$ in response to the changes in $R a, P r, n$ and $r_{i} / L$ and its strong dependence on the choice of initial condition severely limit the usefulness of a correlation for $\overline{N u}_{c y}$, it can be seen from Figs. 4 and 5 that the correlation satisfactorily capture the variation of the mean Nusselt number $\overline{N u}_{c y}$ even for cylindrical enclosures when one convection roll flow pattern is observed. Furthermore, Figs. 4 and 5 suggest that Table 1 can be used to correlate $\overline{N u}_{c y}$ for a wide range of values of nominal Rayleigh and Prandtl numbers considered here except for small values of $r_{i} / L$.

In Rayleigh-Bernard configuration a threshold value of nominal Rayleigh number needs to be surpassed for the onset of fluid motion. For Newtonian (i.e. $n=1$ ) fluids, where the instability is super-critical [1,9], the critical Rayleigh number is estimated by linear regression of $\left(\overline{N u}_{c y}-1\right)$ as a function of $R a$. In the case of shear-thinning fluids, where the instability is sub-critical, the critical Rayleigh number is estimated by reducing nominal Rayleigh number $R a$ in steps of 50 from an established flow condition where $\overline{N u}_{c y} \gg 1$. It is possible to obtain large values 
of viscosity at low shear rate values for shear thinning fluids (i.e. $n<1)$. Thus, $\left(\bar{N}_{c y}-1\right)$ jumps abruptly from zero value with an increase in $R a$ for shear thinning fluids (i.e. $n<1$ ) according to the expectation of sub-critical instability. Table 2 lists the nominal values of Rayleigh number $R a$, above which $\overline{N u}_{c y}$ values deviate from unity, which has been taken for the critical Rayleigh number $R a_{\text {crit }}$ for the onset of convection in this analysis for shearthinning and Newtonian fluids. Table 2 shows that $R a_{\text {crit }}$ decreases with decreasing $n$, which is consistent with previous analyses $[1,12]$. Furthermore, $R a_{\text {crit }}$ values are found to be mostly independent of $r_{i} / L$ and remain equal to the corresponding values for square enclosures except for $r_{i} / L=0$ where slightly smaller values of $R a_{c r i t}$ have been obtained for CWT boundary condition. However, $\left(\overline{N u}_{c y}-1\right)$ cannot be used for a sufficiently accurate measure of the onset of fluid motion for shear-thickening (i.e. $n>1)$ fluids since $\left(\overline{N u} \bar{u}_{c y}-1\right)$ remains close to 0.0 for a large range of $R a$. It has been found that fluid flow initiates for any non-zero value of nominal Rayleigh number by examining the variation of the maximum values of stream function $\psi_{\max }$ with Rayleigh number (not shown here but refer to Fig. $8 \mathrm{~b}$ in Ref. [1]).Thus, the critical Rayleigh number for shear-thickening (i.e. $n>1$ ) fluids can be considered as $R a_{c r i t}=0$. The values of nominal Rayleigh number $R a_{\overline{N u}}{ }_{c y}=1$ for which $\overline{N u}_{c y}=1.001$ for shear thickening (i.e. $n>1$ ) fluids are listed in Table 3 . For shear-thickening fluids viscosity assumes vanishingly small values for small shear rate values for $n>1$ and thus it does not offer any resistance to fluid motion once the nominal Rayleigh approaches zero. This is found to be consistent with previous findings $[1,13]$. It can be seen from Table 3 that $R a_{\overline{N u}} \overline{c y}=1$ remains largely independent of $r_{i} / L$ for both CWT and CWHF boundary conditions but $R a_{\overline{N u}}{ }_{c y}=1$ for $r_{i} / L=0$ has been found to be slightly smaller in the case of CWT boundary condition.

\section{CONCLUSIONS}

The effects of $R a, P r, n, r_{i} / L$ on steady state laminar Rayleigh-Bénard convection of power-law fluids in square cross-sectional cylindrical annular enclosures have been numerically investigated under the assumption of axisymmetry for a range of different values of nominal Rayleigh number $R a$, nominal Prandtl number $P r$, powerlaw index $n$ and the internal radius to enclosure height ratio $r_{i} / L$ (i.e. $10^{3} \leq R a \leq 10^{5} ; 10 \leq \operatorname{Pr} \leq 10^{4} ; 0.6 \leq$ $n \leq 1.8 ; 0 \leq r_{i} / L \leq 24$ ) for both CWT and CWHF boundary conditions. The mean Nusselt number $\overline{N u}_{c y}$ dependence of $\operatorname{Ra}, \operatorname{Pr}, n$ and $r_{i} / L$ shows complex non-monotonic trend due to changes in flow pattern (i.e. number of cells) for shear thinning fluids in the CWT configuration. However, this behaviour is much weaker for shearthickening fluids. Moreover, $R a, P r, n$ and $r_{i} / L$ dependences of $\overline{N u}_{c y}$ have been found to be strongly affected by the choice of initial condition. It has also been found that the mean Nusselt number $\overline{N u}_{c y}$ assumes higher values for CWT boundary condition than that in the case for CWHF boundary condition for large (small) values of $R a$ $(n)$ in the flow regime where the qualitative nature of the flow pattern remains unchanged, whereas an opposite trend is observed for small (large) values of $R a(n)$. Additionally, the critical Rayleigh number $R a_{c r i t}$ for the onset of convection is found to be largely independent of $r_{i} / L$ for both CWT and CWHF boundary conditions.

\section{ACKNOWLEDGEMENTS}

The help provided by Alex Powell Davies is gratefully acknowledged.

\section{REFERENCES}

[1] S. Yigit, R.J. Poole, N. Chakraborty, Aspect ratio effects on laminar Rayleigh-Bénard convection of powerlaw fluids in rectangular enclosures: A numerical investigation, Int. J. Heat and Mass Trans. 91(2015) 292-1307. [2] M. Lamsaadi, M. Naïmi, M. Hasnaoui, Natural convection of non-Newtonian power law fluids in a shallow horizontal rectangular cavity uniformly heated from below, Heat Mass Trans. 41(2005) 239-249.

[3] M. Ohta, M. Akiyoshi, E. Obata, A numerical study on natural convective heat transfer of pseudo-plastic fluids in a square cavity, Num. Heat Trans. Part A 41 (2002) 357-372.

[4] H. Inaba, C. Daib and A. Horibe, Natural convection heat transfer of micro-emulsion phase-change material slurry in rectangular cavities heated from below and cooled from above, Int. J. Heat Mass Trans. 46(2003) 44274438.

[5] G. B. Kim, J.M. Hyun, H. S. Kwak, Transient buoyant convection of a power law non-Newtonian fluid in an enclosure, Int. J. Heat Mass Trans. 46(2003) 3605-3617.

[6] O. Turan, F. Fotso-Choupe, J. Lai, R.J. Poole and N. Chakraborty, Boundary condition effects on laminar natural convection of power-law fluids in a square enclosure heated from below with differentially heated horizontal walls, Ind. Eng. Chem. Res. 53(2014) 456-473. 
[7] S. Yigit, T. Graham, R. J. Poole, N. Chakraborty, Numerical investigation of steady-state laminar convection of power-law fluids in square cross-sectioned cylindrical annular cavity with differentially-heated vertical walls, Int. J. Num. Heat and Fluid Flow 26(2016) 85-107.

[8] S. Yigit, J. Ford, R. J. Poole, N. Chakraborty, Numerical Investigation of boundary condition effect on laminar natural convection of power-law fluids in square cross-sectional cylindrical annular space with differentially heated vertical walls, Comput. Therm. Sci. 7(2015) 261-282.

[9] D. Venturi, X. Wan, G. Karniadakis, Stochastic bifurcation analysis of Rayleigh-Bénard convection, J. Fluid Mech. 650(2010) 391-413.

[10] B. Albaalbaki, R.E. Khayat, Pattern selection in the thermal convection of non-Newtonian fluids, J. Fluid Mech. 668(2011) 500-550.

[11] A. Bejan, Convection Heat Transfer, John Wiley Sons Inc., New York (1984).

[12] C. Tien, H.S. Tsuei \& Z.S. Sun, Thermal convection of a horizontal layer of non-Newtonian fluid heated from below, Int. J. Heat Mass Transfer 12(1969) 1173-1178.

[13] Z. Alloui, N. Ben Khelifa, H. Beji, P. Vasseur, A. Guizani, The onset of convection of power-law fluids in a shallow cavity heated from below by a constant heat flux, J. Non-Newt. Fluid Mech. 196(2013) 70-82.

\section{TABLES}

Table 1: The correlations for natural convection of power-law fluids in square enclosures (i.e. $L / r_{i}=0$ ) proposed by Turan et al. [6].

\begin{tabular}{|c|c|}
\hline CWT & $\begin{array}{l}\overline{N u}_{c y}=\overline{N u}_{1} \text { when } \overline{N u}_{1}=0.178 R a_{C W T}{ }^{0.019} \frac{P r^{0.27}}{(1+P r)^{0.02}}\left(\frac{R a_{C W T^{2-n}}}{P r^{n}}\right)^{1 /(2(n+1))} A>1 \\
A=\exp \left[(n-1) c_{1} R a_{C W T} c_{2} P^{c_{3}}\right] \text { for } n \leq 1 \\
c_{1}=0.994, c_{2}=0.091 \text { and } c_{3}=0.051 \text { for } 5 \times 10^{3} \leq R a_{C W T} \leq 1 \times 10^{5} \\
A=n^{d} \text { for } n>1 \\
d=\left(0.19 P r^{0.04} \ln R a\right) \text { for } 5 \times 10^{3} \leq R a_{C W T} \leq 1 \times 10^{4} \\
d=\left(0.23 P r^{0.04} \ln R a\right) \text { for } 1 \times 10^{4}<R a_{C W T} \leq 1 \times 10^{5} \\
\overline{N u}_{c y}=1 \text { when } \overline{N u}_{1} \leq 1\end{array}$ \\
\hline CWHF & $\begin{array}{l}\overline{N u}_{c y}=\overline{N u}_{2} \text { when } \overline{N u}_{2}=0.289 R a_{C W H F}^{0.014} \frac{P r^{0.217}}{(1+P r)^{0.017}}\left(\frac{R a_{C W H F}{ }^{1-n / 2}}{P r^{n / 2}}\right)^{1 /(n / 2+2)} A>1 \\
A=\exp \left[(n-1) c_{1} R a_{C W H F} c_{2} \operatorname{Pr}^{c_{3}}\right] \text { for } n \leq 1 \\
c_{1}=0.345, c_{2}=0.129 \text { and } c_{3}=0.103 \text { for } 5 \times 10^{3} \leq R a_{C W H F} \leq 1 \times 10^{5} \\
A=n^{d} \text { for } n>1 \\
d=\left[0.136 \operatorname{Pr}^{0.04} \ln R a\right]^{1.5} \text { for } 5 \times 10^{3} \leq R a_{C W H F} \leq 1 \times 10^{4} \\
d=\left[0.145 \operatorname{Pr}^{0.04} \ln R a\right]^{1.5} \text { for } 1 \times 10^{4}<R a_{C W H F} \leq 1 \times 10^{5} \\
\overline{N u}_{c y}=1 \text { when } \overline{N u}_{2} \leq 1\end{array}$ \\
\hline
\end{tabular}

Table 2. Values of critical Rayleigh number $R a_{\text {crit }}$ for different values of $n$ and $r_{i} / L$ for both CWT and CWHF boundary conditions at $\mathrm{Pr}=10^{3}$. Here $R a_{c r i t}$ is estimated by linear regression for Newtonian and reducing $R a$ in steps of 50 for shear-thinning (i.e. $n<1$ ) fluids from an established flow condition where $\overline{N u}_{c y} \gg 1$. The critical Rayleigh number is equal to zero for the shear thickening (i.e. $n>1$ ) fluids.

\begin{tabular}{|c|c|c|c|c|c|c|c|c|}
\hline \multirow{3}{*}{$\boldsymbol{n}$} & \multicolumn{4}{|c|}{ CWT } & \multicolumn{4}{c|}{ CWHF } \\
\cline { 2 - 9 } & \multicolumn{4}{|c|}{$\boldsymbol{r}_{\boldsymbol{i}} / \boldsymbol{L}$} & \multicolumn{4}{c|}{$\boldsymbol{r}_{\boldsymbol{i}} / \boldsymbol{L}$} \\
\cline { 2 - 9 } & 0 & 1 & 16 & Square & 0 & 1 & 16 & Square \\
\hline $\mathbf{0 . 6}$ & 750 & 800 & 800 & 800 & 650 & 650 & 650 & 650 \\
\hline $\mathbf{0 . 8}$ & 1400 & 1550 & 1550 & 1600 & 1150 & 1150 & 1150 & 1150 \\
\hline $\mathbf{1}$ & 2250 & 2600 & 2600 & 2600 & 1700 & 1700 & 1700 & 1700 \\
\hline
\end{tabular}


Table 3: Values of Rayleigh number $R a_{\overline{N u}}=1$ for which $\overline{N u}_{c y}$ values deviate from unity in the third decimal place (i.e. $\overline{N u}_{c y} \approx 1.001$ ) for shear thickening fluids or both CWT and CWHF boundary conditions at $\operatorname{Pr}=10^{3}$.

\begin{tabular}{|c|c|c|c|c|c|c|c|c|}
\hline \multirow{3}{*}{$n$} & \multicolumn{4}{|c|}{ CWT } & \multicolumn{4}{c|}{ CWHF } \\
\cline { 2 - 9 } & \multicolumn{4}{|c|}{$\boldsymbol{r}_{\boldsymbol{i}} / \boldsymbol{L}$} & \multicolumn{4}{c|}{$\boldsymbol{r}_{\boldsymbol{i}} / \boldsymbol{L}$} \\
\cline { 2 - 9 } & 0 & 1 & 16 & Square & 0 & 1 & 16 & Square \\
\hline $\mathbf{1 . 4}$ & 3250 & 3500 & 3500 & 3500 & 2000 & 2000 & 2000 & 2000 \\
\hline $\mathbf{1 . 8}$ & 5000 & 5000 & 5000 & 5000 & 2500 & 2500 & 2500 & 2500 \\
\hline
\end{tabular}

\section{FIGURE CAPTIONS}

Fig. 1: Schematic diagram of simulation domain: a) CWT, b) CWHF configuration.

Fig. 2: Variation of $\overline{N u}_{c y}$ with $\operatorname{Pr}$ for different values of $R a, n$ and $r_{i} / L$ for CWT boundary condition for both E.F and Q.F initial conditions.

Fig. 3: Variation of $\overline{N u}_{c y}$ with $P r$ for different values of $R a, n$ and $r_{i} / L$ for CWHF boundary condition for both E.F and Q.F initial conditions.

Fig. 4: Variation of $\overline{N u}_{c y}$ with $r_{i} / L$ (for $r_{i} / L \leq 1.0$ ) for different values of $R a$ and $n$ at $\operatorname{Pr}=10^{3}$ for both CWT and CWHF boundary conditions in the cases of E.F and Q.F initial conditions. The corresponding values of mean Nusselt number for square enclosures (i.e. $\overline{N u}_{s q}$ ) are shown with broken lines. The correlation results for $\overline{N u}_{s q}$ in Table 1 are shown by solid lines.

Fig. 5: Variation of $\overline{N u}_{c y}$ with $r_{i} / L$ (for $r_{i} / L>1.0$ ) for different values of $R a$ and $n$ at $P r=10^{3}$ for both CWT and CWHF boundary conditions in the cases of E.F and Q.F initial conditions. The corresponding values of mean Nusselt number for square enclosures (i.e. $\overline{N u}_{s q}$ ) are shown with broken lines. The correlation results for $\overline{N u}_{s q}$ in Table 1 are shown by solid lines. 


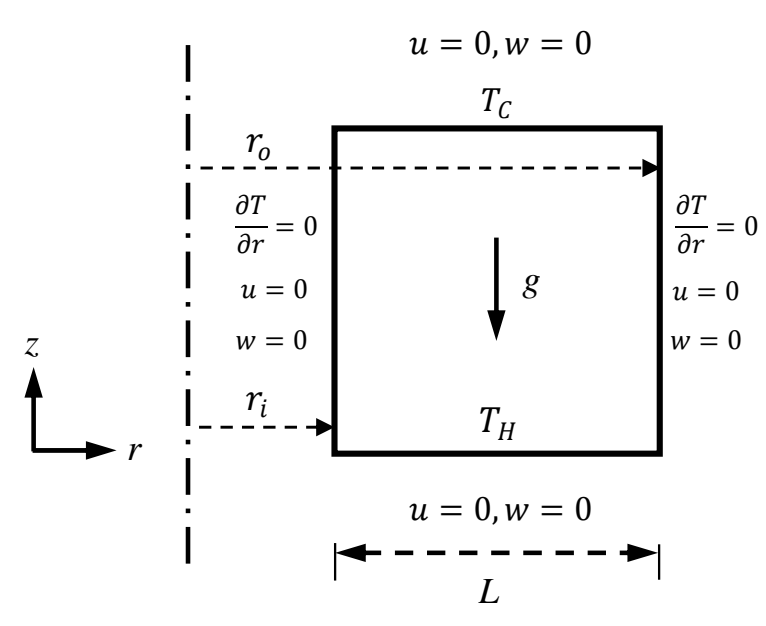

a)

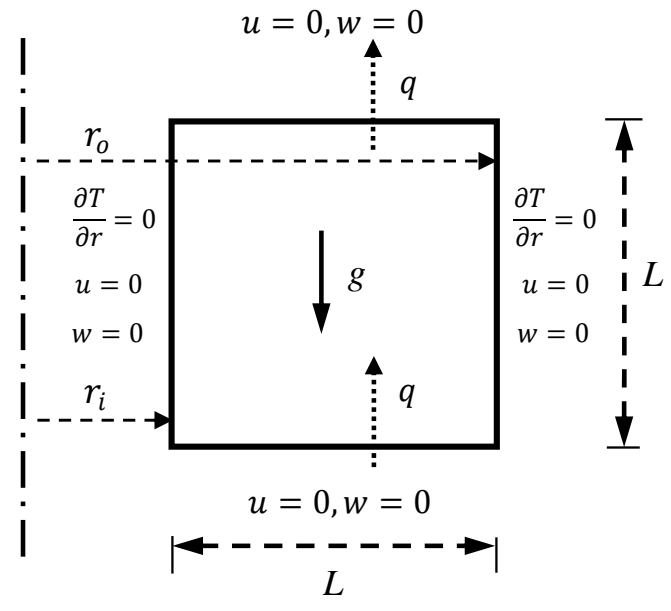

b)

Fig. 1: Schematic diagram of simulation domain: a) CWT, b) CWHF configuration. 

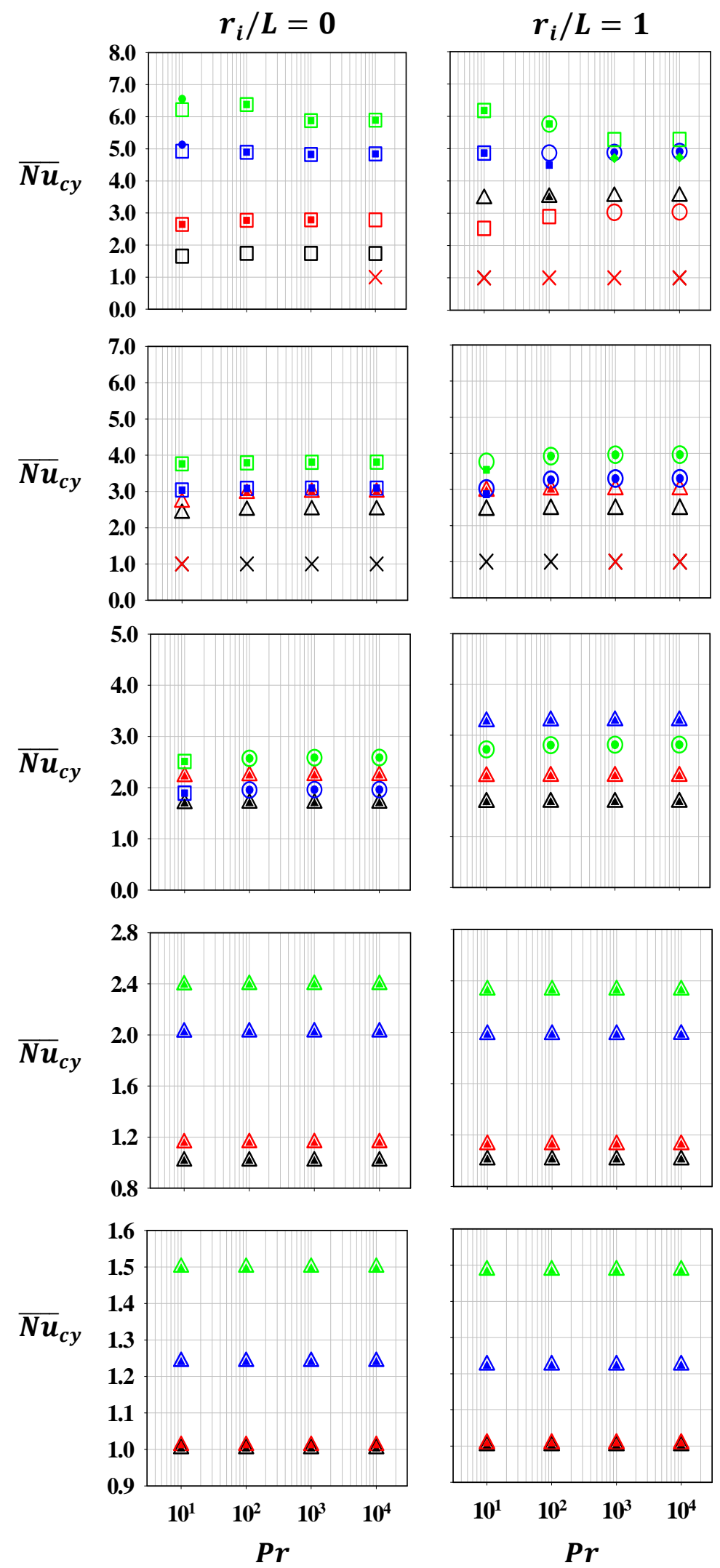
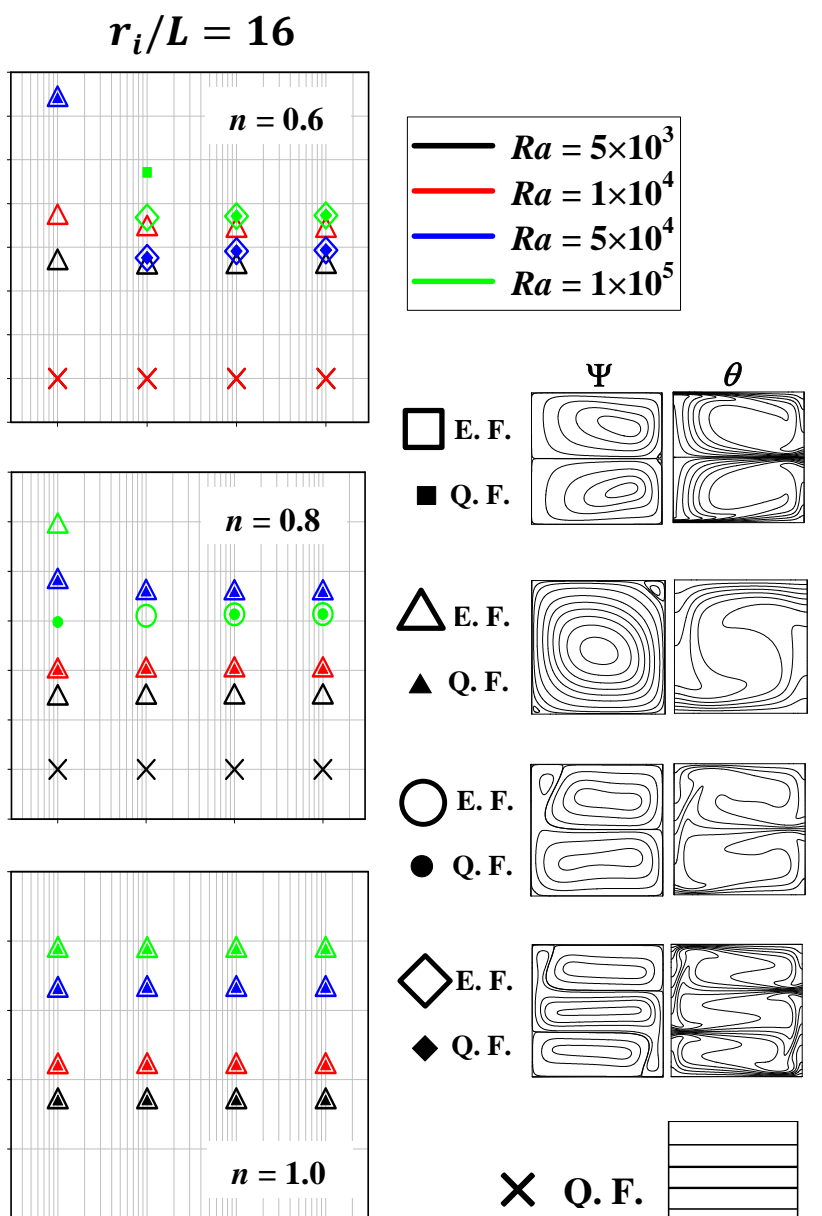

X Q. F.
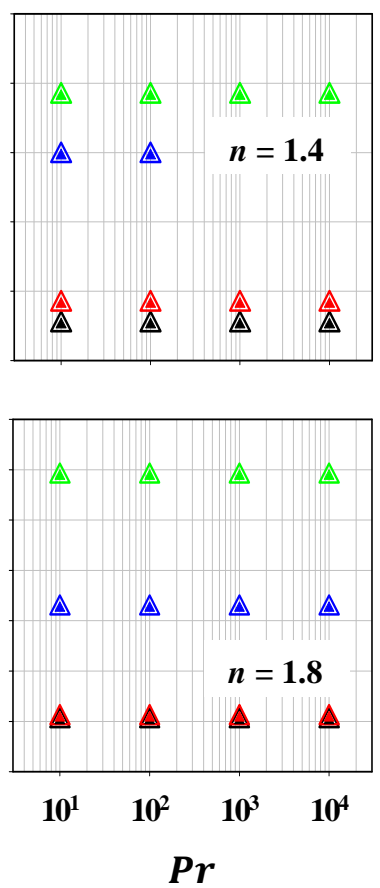

Fig. 2: Variation of $\overline{N u}_{c y}$ with $\operatorname{Pr}$ for different values of $R a, n$ and $r_{i} / L$ for CWT boundary condition for both E.F and Q.F initial conditions. 


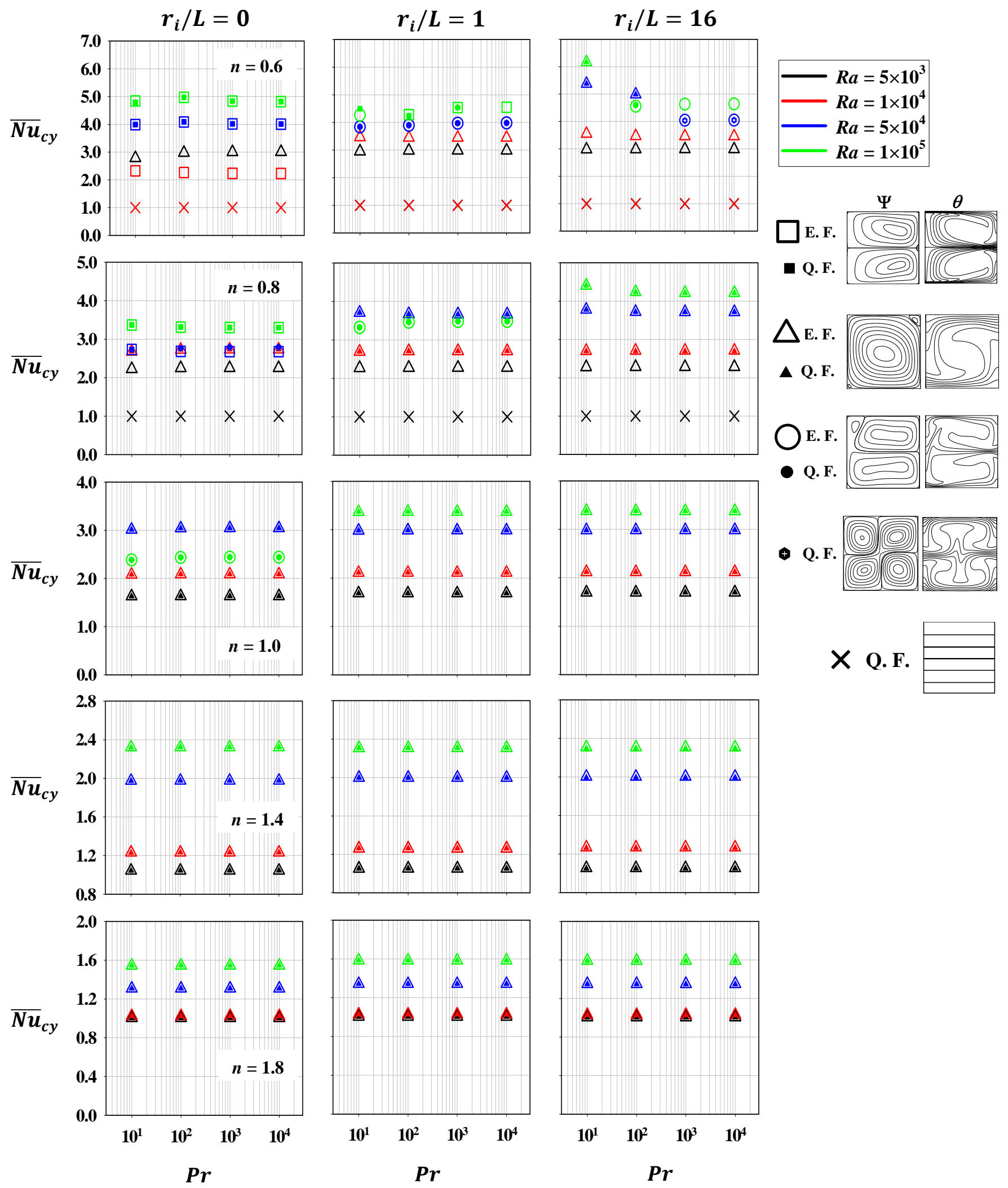

Fig. 3: Variation of $\overline{N u}_{c y}$ with $\operatorname{Pr}$ for different values of $R a, n$ and $r_{i} / L$ for CWHF boundary condition for both E.F and Q.F initial conditions. 


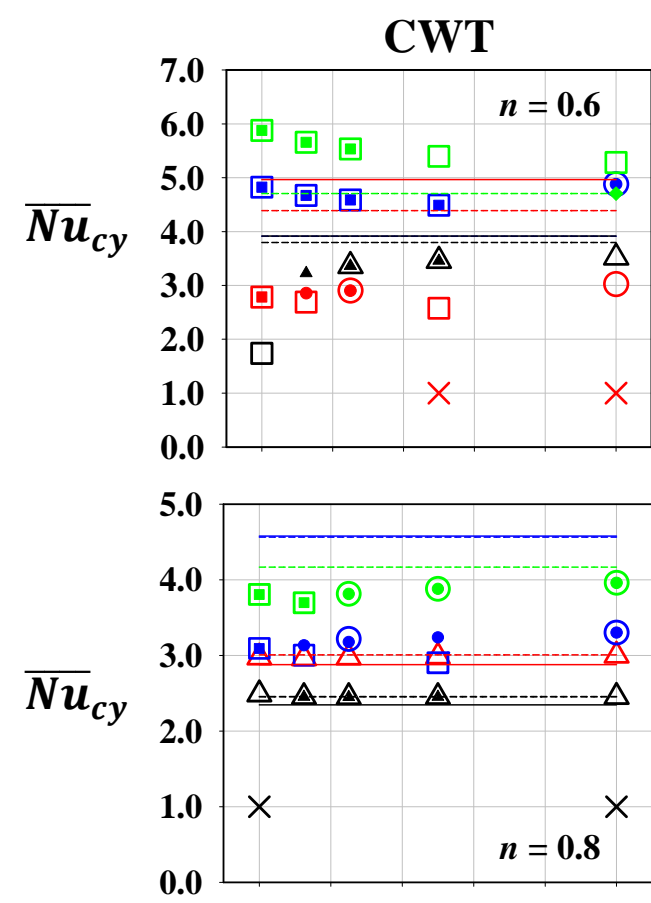

\section{CWHF}
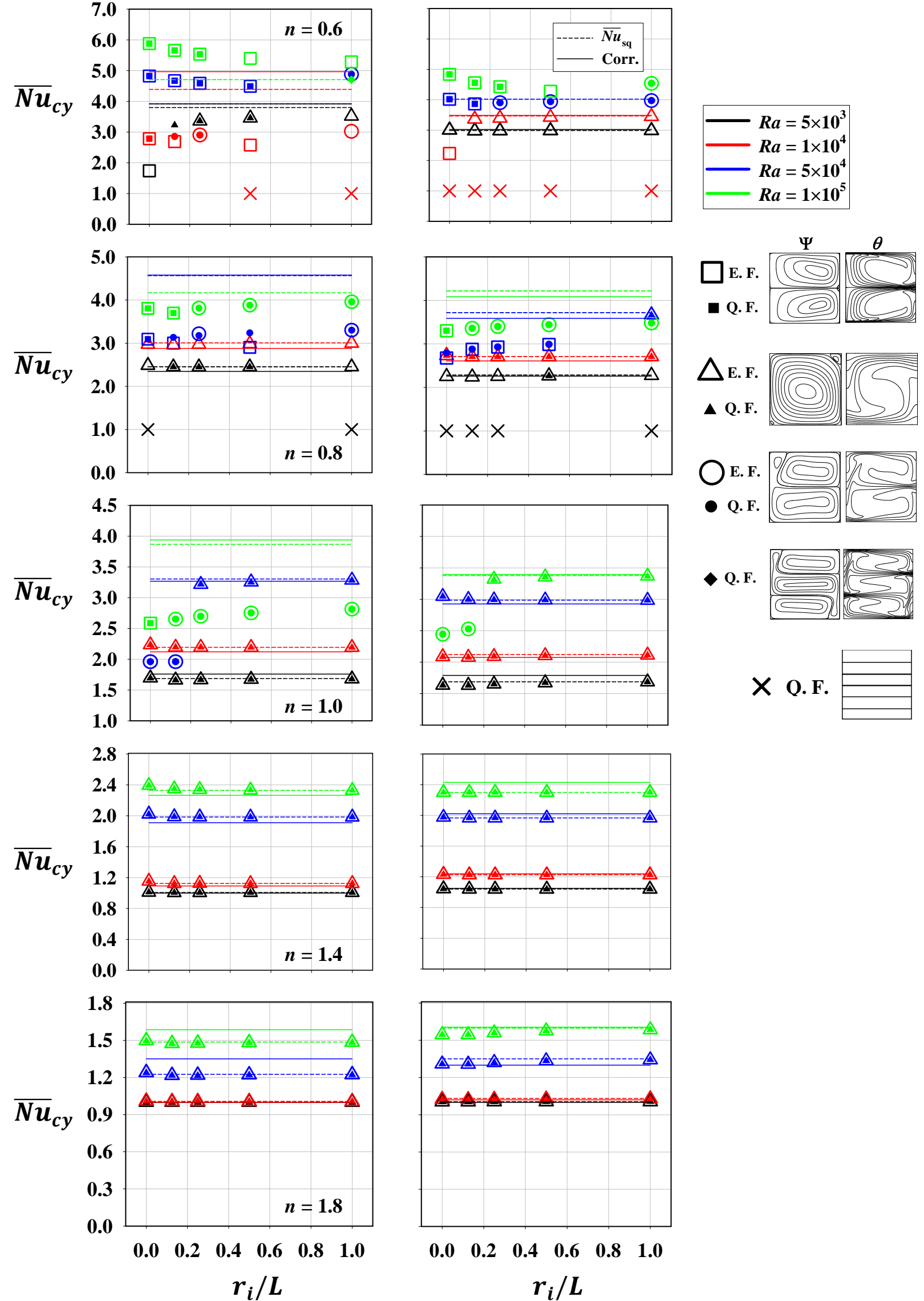

Fig. 4: Variation of $\overline{N u}_{c y}$ with $r_{i} / L$ (for $r_{i} / L \leq 1.0$ ) for different values of $R a$ and $n$ at $P r=10^{3}$ for both CWT and CWHF boundary conditions in the cases of $\mathrm{E} . \mathrm{F}$ and Q.F initial conditions. The corresponding values of mean Nusselt number for square enclosures (i.e. $\overline{N u}_{s q}$ ) are shown with broken lines. The correlation results for $\overline{\boldsymbol{N u}}_{s q}$ in Table 1 are shown by solid lines. 

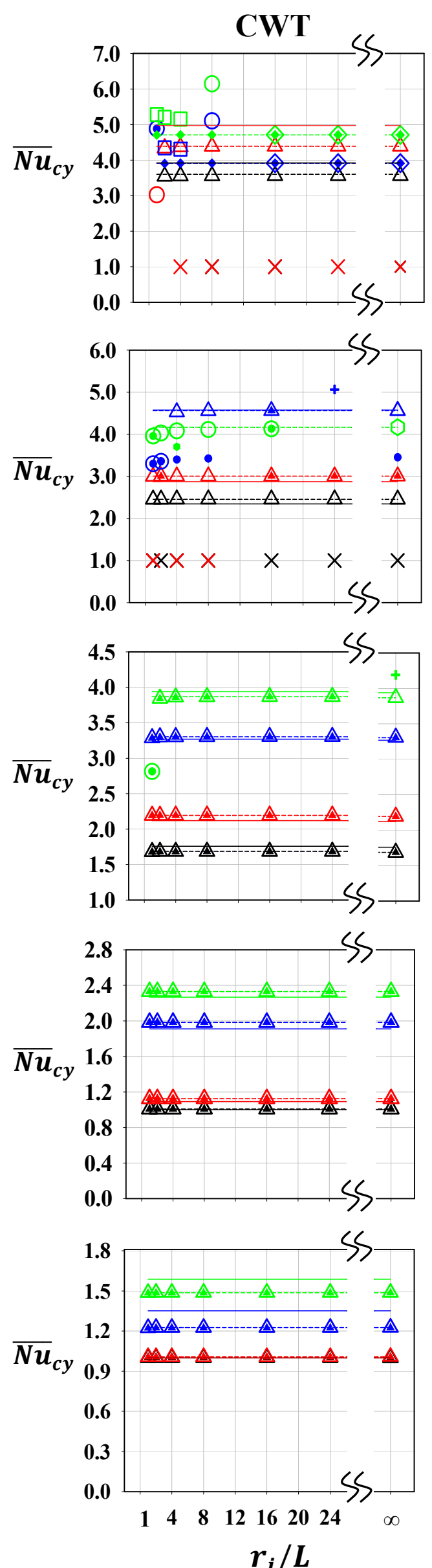

CWHF
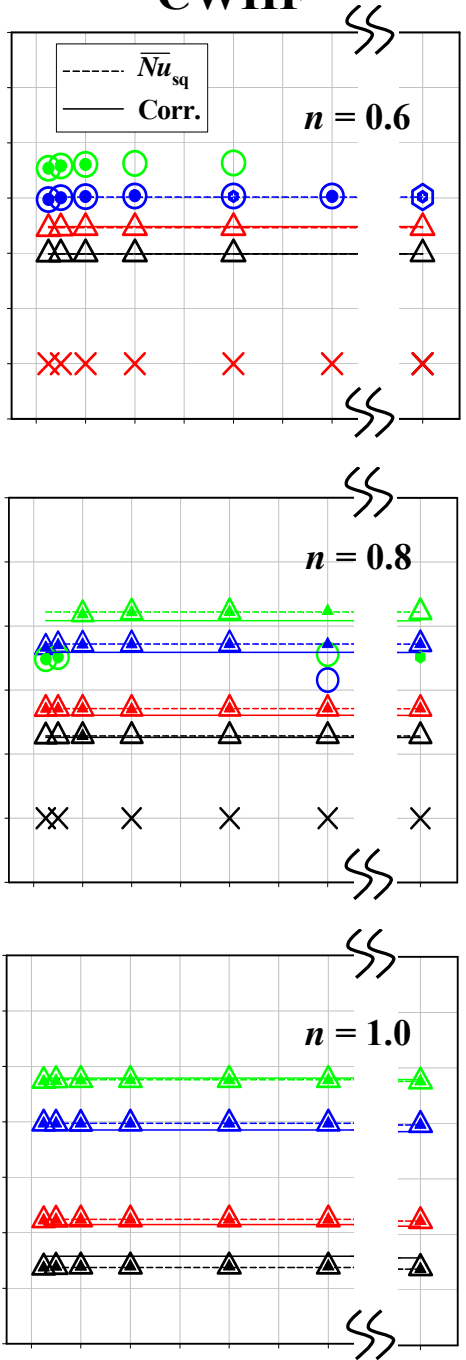

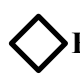

$\triangle$ E. F.

$\Delta$ Q.F.
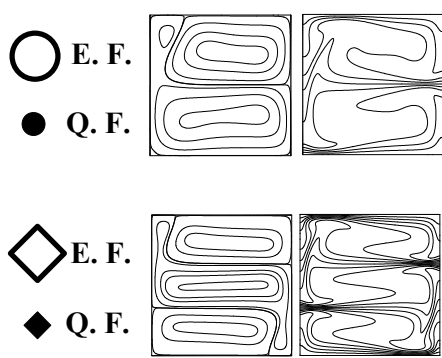

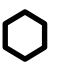

E. F.

Q.F.

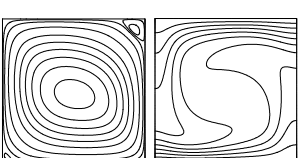

SS
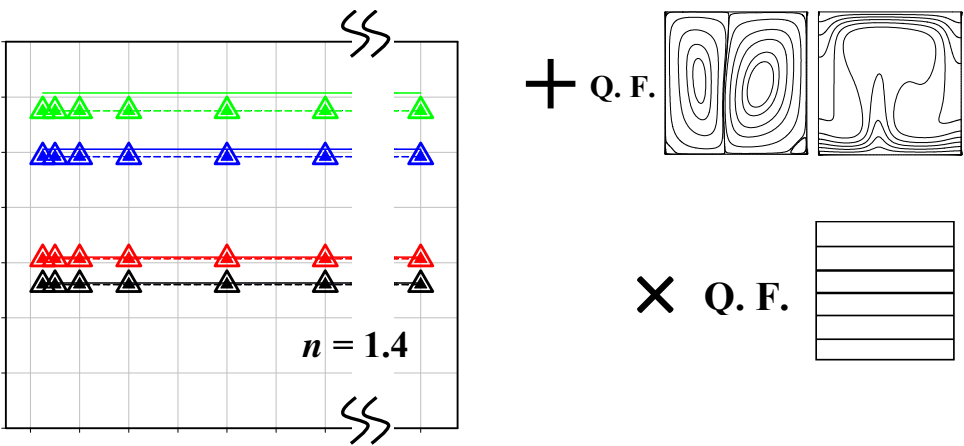

X Q. F.

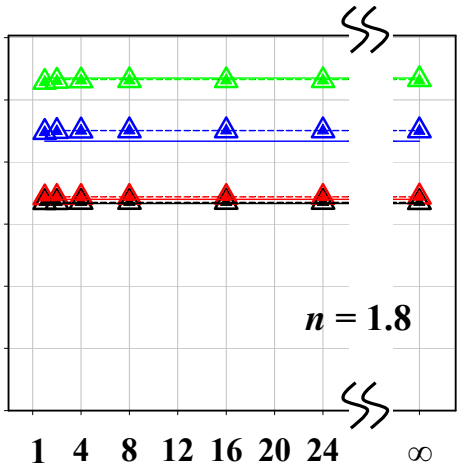

$\boldsymbol{r}_{i} / L$

Fig. 5: Variation of $\overline{N u}_{c y}$ with $r_{i} / L$ (for $r_{i} / L>1.0$ ) for different values of $R a$ and $n$ at $P r=10^{3}$ for both CWT and $C W H F$ boundary conditions in the cases of $E$.F and Q.F initial conditions. The corresponding values of mean Nusselt number for square enclosures (i.e. $\overline{N u}_{s q}$ ) are shown with broken lines. The correlation results for $\overline{N u}_{s q}$ in Table 1 are shown by solid lines. 